\title{
THE MATHEMATICS OF COMPUTING BETWEEN LOGIC AND PHYSICS
}

\author{
GIUSEPPE LONGO AND THIERRY PAUL
}

\begin{abstract}
Do physical processes compute? And what is a computation? These questions have gained a revival of interest in recent years, due to new technologies in physics, new ideas in computer sciences (for example quantum computing, networks, non-deterministic algorithms) and new concepts in logic. In this paper we examine a few directions, as well as the problems they bring to the surface.
\end{abstract}

\section{Contents}

1. Introduction 2

2. Computability and continuity 3

3. Mathematical computability and the reality of physics 6

4. From the principle of least action to the quantum theory of fields 8

5. Chaotic determinism and predictability 9

6. Return to computability in mathematics 14

$\begin{array}{ll}\text { 7. Non-determinism? } & 16\end{array}$

8. The case of quantum mechanics 19

9. Randomness, between unpredictability and chaos 22

10. General conclusions 24

$\begin{array}{ll}\text { References } & 27\end{array}$

Département d'Informatique UMR 8548 et CNRS, longo@ di.ens.fr

Département de Mathématiques et Applications.UMR 8553 et CNRS, paul@ dma.ens.fr

École Normale Supérieure, 45, rue d'Ulm - F 75730 Paris cedex 05

This is a largely expanded version of a previous paper in French to appear in the proceedings of the LIGC colloquium, Cerisy, September 2006 (Joinet et al., eds), Hermann, 2008 (see http://www-philo.univ-paris1.fr/Joinet/ligc.html). 


\section{INTRODUCTION}

Digital machines, by their extraordinary logical and computational capability, are changing the world. They are changing it with their power and their originality, but also with the image of the world they reflect: they help perform thousands of tasks and enable radically new ones, they are an indispensable tool for scientific research, but they also project their own mathematical structure upon the processes they are involved in. These machines are not neutral, but are in fact extremely original and have a complex history, based on several turning points in terms of the thinking which enabled to invent them. They synthesize a vision and a science which is very profound. They are "alphabetic" in the specific sense of the encoding of human language, produced by a bagpipe over strings, by means of discrete and meaningless letter-units, an incredible invention which dates back 5,000 years. They are Cartesian in their software/hardware duality and in their reduction of thought to the elementary and simple steps of arithmetic calculus. They are logical by stemming from a logico-arithmetical framework, in the tradition of Frege and Hilbert, during the 30s ("proofs are programs"). And this by the final remarkable invention, by Gödel: the number-theoretic encoding of any alphabetic writing. For all of these reasons, they contribute to a reading of nature based on the computable discrete, from the alphabet to arithmetic, on a space-time framed within discrete topology, of which the access and the measurement are exact, just like in digital databases.

We will see why confounding physics, despite its great "mathematicity", with calculus, in any form whatsoever, seems as a mistake to us. First, the idea that physics "reduces to solving" equations is a mistaken idea. To be assured of this, one needs only to consider that a great part of physics concerns variational problems in which the search of a geodesic differs greatly from the search for the solution to an equation, without mentioning the singular quantum situation, to be discussed below, nor the life sciences, which are not very mathematized and for which the notions of invariant and of the transformation which preserves it, central to mathematics, are far from being "stabilized".

The new importance of digital machines, in particular in the natural sciences, requires a thorough analysis of the relationship between computations and natural processes. We will focus here on the relationships between computations and, among the physical processes, those which we consider as "natural", that is, those that occur "independently" of human intervention (because a machine also produces, or even is, a physical process, but it is a result of a human construction which is extremely original and theoretically rich). We will then ask the question: Do physical processes compute? 


\section{Computability And COntinuity}

The naive, and unfortunately highly widespread response to this question is that yes, everything can be seen in terms of alphanumeric information and its computational elaboration. This is reminiscent of the age of the clock, when everything was clockwork - and when iatromechanics was inventing modern anatomy, though working on the physiology of hydraulic pumps, the medicine of bloodletting. This thesis, under different forms, is often called the "physical Church thesis". So let's return briefly to this thesis in its original form, which is purely logico-mathematical and in no way physical.

Church's thesis, introduced in the 30s after the functional equivalence proofs of various formal systems for computability (and concerning only computability over integers), is an extremely solid thesis: it ensures that any finitistic formal system over integers (a Hilbertian-type logico-formal system) computes at best classical recursive functions, as defined by Gödel, Kleene, Church, Turing.... This thesis therefore emerged within the context of mathematical logic, as grounded on formal systems for arithmetic/discrete computations: the lambda-calculus (Church, 1932), a system for the functional encoding of logical deductions, and Turing's Logical Computing Machine ${ }^{1}$, were the motors of various equivalence proofs ${ }^{2}$.

The very first question to ask is the following: If we broaden the formal framework, what happens? For example, if we consider as basic support for computation a set "greater" than the natural integers, is this invariance of formalisms preserved? Of course, if we want to refer to continuous (differentiable) physics-mathematics, an extension to consider may be the following: What about the computational processing of these computable "limit" numbers which are computable real numbers? Are the various formalisms for computability over real numbers equivalent, when they are maximal? An affirmative response could suggest a sort of Church thesis "extended" to this sort of computational "continuity". Of course, the computable reals are

\footnotetext{
${ }^{1}$ 1936: "A man provided with paper, pencil and rubber, and subject to a strict discipline, is in effect a Universal (Turing) Machine", [29]. In fact, the reader/writer needs only to know how to read/write 0 and 1 on an endless length of tape, then to move one notch to the right or to the left, according to given instructions (write, erase, move right, move left) to compute any formally computable function (see the next note).

${ }^{2}$ The other definitions of computability are more "mathematical": they propose, in different ways, arithmetic function classes which contain the constant function 0 , the identity and the successor functions +1 , and which are closed by composition, by recursion (in short: $f(x+1)=h(f(x), x)$ ) and by minimization (that is, $f(x)=\min _{y}[g(x, y)=0]$ ). It is a mathematically non obvious remark that by reading/writing/moving 0 s and $1 \mathrm{~s}$ left and right on a tape it is possible to calculate all of these functions: there lies the genius of Turing and the origin of the 0 and 1 machine which will change the world.
} 
countably many, but they are dense in the "natural" topology over Cantor's reals, a crucial difference as we shall see.

With this question, we then begin to near physics, all the while remaining in a purely mathematical framework, because mathematics on the continuum of real numbers constitutes a very broad field of application to physics, since Newton and Leibniz. In particular, it is within spatial and often also temporal continuity that we represent dynamical systems, that is, most mathematical models (in logical terms: mathematical formalisms) for classical physics. This does not imply that the world is continuous, but only that we have said many things thanks to continuous tools as very well specified by Cantor (but his continuum is not the only possible one: Lawvere and Bell, say, [6], proposed another without points, but one which is unfortunately not richer for the moment in mathematical terms - although some may hope to use it to better address the geometry of quantum physics; so, let's rest on Cantor for the time being).

Now, from this equivalence of formalisms, at the heart of Church's thesis, there remains nothing regarding computability over real numbers: the models proposed, in their original structure, are demonstrably different, in terms of computational expressiveness (the classes of defined functions).

Today, it is possible to roughly group different formal systems into three main groups (however not exhaustive ones), in order to perform computations over real numbers:

- recursive analysis, which develops the approach to Turing's computable real numbers, or even the Turing Machine itself, by an infinite extension recently formalized by Weihrauch (two tapes, one which can encode a computable real hence infinite number, and the other which encodes the program, see [33]; from the mathematical standpoint, the idea was first developed by Lacombe and Grezgorzcyk, in 1955-57);

- the Blum, Shub and Smale BSS model (an infinite tape and a little control system, see [7]);

- the Moore-type recursive real functions (defined in a more mathematical manner: a few basic functions, and closure by composition, projection, integration and search for the zero, see [22]);

- different forms of "analog" systems, among which threshold neurons, the GPAC (General Purpose Analog Computer, attributable to Shannon, [28], of which a first idea preceded classical recursivity: V. Bush, M.I.T., 1931, [10]).

Each of these systems has its own interest. Besides they confirm the solidity of Church's original thesis, since the restriction to integers of all known models of computable continuity again produces classical recursivity (or no 
more than that). What else could we say, concerning inclusions, links, demonstrable passages, as for these formalisms for computability on continua?

Of course, it is relative continuity: computable real numbers do not form a Cantor-type continuum, as we said; they are a denumerable set of measure 0. However, their "natural" topology is not the discrete topology (and mathematicians know what "natural" means: the discrete topology over Cantor reals is not natural, one does nothing with it). This is the crucial mathematical difference from computability over the isolated points of the countable discrete.

The difference is crucial with regard to physical modelization for the following reasons. In physics, the (Cartesian) dimension of space is fundamental: relativity and string theory, to use some examples, make it into a constitutive issue; but also, the propagation of heat, or the mean field theory, to remain in classical physics, depend in an essential way upon the dimension under consideration, see [3]. Now, computability over real numbers is "indifferent" to the Cartesian dimension: one cannot change the expressivity of the machine by changing the dimensions of its databases, but only the polynomial efficiency. This is due to the computable isomorphism $<., .>$ between $N^{2}$ and $N$. One may therefore define, without difficulty and for any discrete formalism, the universal function $U$ within the very class of computable functions (that is, once the computable functions have been enumerated, $\left(f_{i}\right)_{i \in N}$, function $U(i, n)=f_{i}(n)$ belongs to such class by the coding $<., .>$ ).

These properties, quite interesting, are a consequence of the rather general fact that discrete topology does not force a dimension. In short, in the discrete universe (the category of sets), any infinite set (real numbers, in particular) is isomorphic to all of its finite (Cartesian) products. But when discrete topology is no longer "natural", within a continuum say, with Euclidean (or real) topology, for example, the spaces having different dimensions are no longer isomorphic. We then say that the dimension is a topological invariant, for topologies which derive from the interval of physical measurement (Euclidean, typically). A remarkable relationship between geometry and physics: the metrics (and the topology induced) of the sphere (or interval) indeed corresponds to the "natural" physical measurement, that of the intervals, and it "forces" the dimension, a crucial notion in physics. So here is a fundamental difference for continuous mathematics (and for computability over continua, would they be just dense): any bijective encoding of spaces with different dimensions is necessarily non-continuous and, in order to define, typically, the universal function, it is necessary to change dimension, hence to leave the given class. 
So let's return to our question, which is, in our view, a rigorous way to address the extensions of the Church thesis to the mathematics of physics: can we correlate different formalisms for computability over a continuum, these being adequate for physical systems and which therefore make the Cartesian dimension into a fundamental issue, even if they are non-equivalent? There are no extensions today of the Church thesis to computable continua and just partial answers are provided by many authors: $[19,9]$ present an overview and recent results which, by the addition of functions and operators which are highly relevant from the physical standpoint, enable to establish inclusions under certain conditions, these being rather informative links. On the basis of these works, we should arrive at a notion of "standard system" for computability over the set of computable real numbers which represent a reasonable extension of Church's thesis to computable continuity (all "standard" systems would be equivalent, modulo the fundamental issue of dimensions), and therefore also find an interesting link with the mathematics of physics.

However, for a large enough class, this standardization is not obvious and we are far from having a Church-like equivalence between systems. Moreover, it is clear that we remain, as in the case of the logico-formal Church thesis, within mathematical formalisms ${ }^{3}$. And what about physical processes?

\section{Mathematical COMPUtability AND the REALity of PHysics}

Let's ask a preliminary question to asking if nature computes: what could nature actually compute? If we look at the object before looking at the method, things may not be so simple. Vladimir Arnol'd recalls in his book [32] the formula attributed to Newton : "It is necessary to solve equations". From another perspective, physics could very well be expressed according to another formula, provided this time by Galileo ${ }^{4}$. And from Galileo's standpoint which is, however, far from being formalistic or number-theoretic but

\footnotetext{
${ }^{3}$ In what concerns the extension of the Church thesis to computer networks and to concurrent systems in general, systems which are perfectly discrete but distributable over space-time, this being better understood by means of continuous tools, we refer to [1] and to its introduction: in this text, it is noted that this thesis, in such a context, is not only false, but also completely misleading (the processes are not input-output relationships and their "computational path" - modulo homotopy, for instance - is the true issue of interest).

4 "La filosofia scritta in questo grandissimo libro che continuamente ci sta aperto innanzi agli occhi (io dico l'Universo) non si puó intendere se prima non s'impara a intender la lingua, e conoscer i caratteri, nei quali è scritto. Egli è scritto in lingua matematica, e i caratteri son triangoli, cerchi, ed altre figure geometriche, senza i quali mezzi è impossibile a intenderne umanamente parola; senza questi è un aggirarsi vanamente per un oscuro laberinto." Il saggiatore, 1623.
} 
rather "geometric", and which continues to perceive "filosofia" as an intermediary between ourselves and the world, the question asked above could very well be natural.

Newton-Arnold's view point seems more modern. However, it is now necessary to observe that the importance of an equation, or more generally, of a mathematical conceptual structure used in physics is often more important in abstracto than the solutions which it proposes. But let's nevertheless look at what happens upwards to this.

\section{Is there something to solve, to compute?}

The description of a physical phenomenon takes place within a framework of "modelization", that is, within a fundamentally "perturbational" framework. That is, the isolation of a phenomenon, its intrinsic comprehension, supposes that we neglect its interaction with the rest of the world. But to neglect does not mean to annihilate: the rest of the world exists and creates perturbations at this isolation. From this point of view, a model must be immersed in an "open set" of models.

The isolation of a concept upon which one is working, for instance, results from the choice of a given scale. Neighboring scales are then supposed to be either inaccessible (smaller scales), or processable by averaging (larger scales). In both cases, they can influence the model and the equation which yields it. Asking the question whether something which we compute, physically, fits into a framework of computability in the sense evoked by this article commands having precautions at least.

In particular, are there equations and only equations? A great part of classical physics rests upon variational principles. The trajectory appears not only as the solution to an equation, but as a solution that is chosen because is optimizes, extremizes a quantity (action). Of course, this is equivalent to resolving equations (Euler-Lagrange), but this is only an equivalency. Let's recall that Feynman [14] preferred solutions to equations for quantum mechanics. In this case, no more equations; all possible trajectories (minimizing or not the action functional) are what should be involved. This is possible, but is so thanks to the functional integral, in an infinite dimension. And what about computability in this case?

Let's look at another example: quantum field theory, a physical theory which is not mathematically well founded yet, but which has been phenomenally successful in terms of precision, is based entirely on perturbative calculations [24].

It is obvious that, even without considering the lack of precision of classical measurement, which we will address later on, the situation is somewhat fuzzy, 
largely perturbative, and hence that the problem of computability in physics is multiple and complex.

But let's suppose that there actually are equations. And let's suppose that the true issue is really the solution, which is predictive. We will then be compelled to remark that the situations where the solution's values are important are rare. A simple example: physicists like to draw curves, even when a formula providing the solution is available. But what is left of computability when the "result" is smooth, where only the general "trends" are important, not the exact values?.

Let's take a look at the dynamical systems provided by maps, the case of the "baker's map", for instance. In principle, there is no mapping in physics; there are fluxes. A map appears when we compute a flux at time 1 (which we will later iterate), but this flux at time 1 is actually computed from equations. The Poincaré first recurrence map, and the dynamical systems which followed, were invented as simpler tools, qualitatively and quantitatively more manageable, but it would be wise to not identify them too much with the initial systems.

In conclusion let us see whether it is possible to consider an isolated equation in physics. As we observed, if equations come in families within which (possibly continuous) parameters change, how must one apprehend the problem of computability, so carefully defined within a discrete and countable space? Maybe nature does compute, but knowledge, our theory of nature, fundamentally rests in huge, infinite spaces (spaces of parametrized equations, typically), which could very well escape any computationalistic approach.

\section{From the PRINCIPle of LEAST ACTION TO THE QUANTUm THEORY OF FIELDS}

The concept of differential equation is not the only one which provides a way of computing dynamics in physics. As we mentioned, an alternative approach consists in minimizing a certain functional (the action) among different candidates for the trajectory. More precisely to any path $\gamma$ going from an initial point to a final one is associated a number, $S(\gamma)$, and the "true" trajectory, the one that effectively is going to follow the particle, is the one which provide the lowest (in fact any extremal) value of these numbers $S(\gamma)$. This principle of "least action" does not ask to solve an equation, it just asks to evaluate the functional $S$ at any possible path $\gamma$, and select the extremal one. If it asks to compute something it doesn't ask to compute a finite number or set of numbers, it asks to evaluate a huge set of numbers, and to find the lowest. 
It is well known that the principle of least action is, in many situations at least, equivalent to the so-called Euler-Lagrange equations, therefore shown to be embedded in the operational setting. But the Feynman "path integral" formulation of quantum mechanics create a revival of this idea of evaluating instead of computing. The quantum amplitude of probability is obtained by summing over all paths (actually the same one we just talked about) expressions of the form $e^{i \frac{S(\gamma)}{\hbar}}$ : the selection to minimizing path disappeared even. Here again this formalism is shown to be equivalent to Schrödinger equation, getting back once more to the operational level.

The situation drastically changes with quantum theory of fields, a mixing of quantum mechanics and partial differential equations. This theory, conceptual basis of our deep understanding of elementary particles, is a generalization of quantum mechanics to infinite dimension. The formalism of quantum theory of fields is an extension of the path integral methods to the case where the "paths" $\gamma$ seat in infinite dimensional spaces. This is the theory which provides nowadays the most accurate numerical agreement with experimental data. It "lives" in an extremely huge space (the space of infinite dimensional paths), and has, up to now, NO equivalent operational setting.

\section{Chaotic Determinism And PRedictability}

In what concerns the relationships between dynamical systems and their capacity to predict physical evolutions, there is often a great confusion between mathematics and physical processes. The notion of deterministic chaotic system is purely mathematical and is given, in a standard way, by three formal properties (see [12]). However, it is legitimate to speak of a physical process and to say that it is deterministic (and chaotic, if such is the case): what is meant by that is that it is possible, or believed to be so, to write a system of equations, or even an evolution function, which determines its evolution (in time or regarding the relevant control parameter).

Unpredictability is then a property which arises at the interface between physical and mathematical processes. One gives oneself a physical process and a mathematical system (a system of equations, or even an evolution function - an iterated system, typically, so a discrete-time system, within a continuous space). Then the process with regard to the system (or even with regard to any reasonable system which we consider to modelize the given process) is said to be unpredictable. A physical process "as such" is not unpredictable: one must attempt to state or even pre-dict, usually by mathematical writing, for there to be unpredictability. Likewise, a mathematical system is not unpredictable, as such: it is written and, if fed values, it computes. 
And this is where computability comes into play. It happens that any "reasonable" mathematical system would be characterized by effective writing: save a pathology (feeding a polynomial with non computable coefficients, Chaitin's omega for instance!), we normally write evolution functions which are computable (we will however see some counter-examples). More specifically, any Cauchy problem (a very broad class of differential equations) admits computable solutions (if solutions there are), in one of the known systems for continuous computability. Interesting pathologies, or counterexamples, do exist; for the moment, it suffices to mention some solutions of the Poisson equation in [25], the boundary of a Julia set, in [5].

But the problem is not only there (not really there, as a matter of fact): the choice of scale, of perturbative method, of phase space (or of hidden variables, or those which were explicitly or unconsciously excluded) shows the constituted autonomy of mathematical language, because mathematics is constructed within a friction contingent to the world and then detaches from it by its symbolic autonomy. And this construction is a highly noncomputable historical decision, often an infinitary transition towards a limit concept. By this, mathematics is not arbitrary, but the result of a constructed objectivity.

In summary, when we write a formalism, we give ourselves something "computable" (grosso modo, because the different continuous systems are not yet unified) but this is obtained by an historical choice or limit process, which singles out the symbolic construction from the world. So the fact of the computability of an evolution function, which we suppose to be adequate regarding the description (modelization) of a physical system, is the evidence which we deduct from its writing. The logistic function, for instance, see $[12,20]$, is a simple and important chaotic system; a computable bilinear function, with a coefficient $k \ldots$ ok, only if we take a non-computable $k$, a crazy choice, it is not.... A very famous variant of the logistic function is also given by continuous but non-differentiable deformation which preserves many of its interesting properties; this is the "tent" function which, grosso modo, modelizes the movements of stretching and mixing of a piece of dough by a baker who is a little stiff and repetitive in his movements. These systems, as in the case of any formal writing, are effective and are in no way unpredictable, as such. We give them values (computable ones) and they compute: within the limits of the available (finite) machine, they produce outputs. However, any physical system which is considered to be modelized (formalized) by one of these functions is unpredictable, even if by one of their non-differentiable variants (an ago-antagonistic system - chemical action-reaction oscillations, for example, or the baker's transformation, 
in the differentiable or non-differentiable case of which we were speaking). As soon as we give the result of a physical measurement, that is, an interval, to the function in question, this interval is mixed and exponentially widened, quickly preventing any prediction of the evolution. Of course, the machine which computes these non-linear functions can also help appreciate chaos:

1 - it provides dense trajectories (sequences of points) in the definition domain;

2 - a difference (at the 16th decimal, for instance) in the numeric input gives very different values after few iterations (about 50 in our logistic cases, see below).

However, if it is re-launched with the same initial values in a discrete context (and this is fundamental) it will always return the same trajectory (sequence of numbers). The point is that, in discrete state machines, access to data is exact: this is the crucial difference w.r. to access to the world by (classical) physical measure, which is given by the interval of (approximated) measurement, by principle (there is at least the thermal fluctuation).

And there lies also the advantage of the discrete state machine, of which the access to the database is exact: it iterates identically, because it is, firstly, an iterating machine. Iteration founds Gödel-type primitive recursion, which is iteration and +1 in a register (see the note above). It enables the portability of the software and hence its identical transferal and iteration at will (and it works - without portability and iterability of software, there would be no computing, nor market for software). You may launch a program hundreds of times, thousands of times and it iterates.

Computer scientists are so good that they have been able to produce reliable and portable software (that is iterating identically) even for networks of concurrent computers, embedded in continuous space-time, with no absolute clock. Yet, the discrete data types allow this remarkable performance. Note that identical iteration of a process is very rare in Nature (fortunately, otherwise we would still be with the Universe of the origin or the early protozoans). We, the humans, along our history, invented the discrete state machines, which iterate. A remarkable human construction, in our space of humanity, using the alphabet, Descartes dualism (software/hardware), Hilbert's systems, Gödel's numberings, Turing's ideas... and a lot of discrete state physics. Computing, programs and alike are not "already there" in nature. Unfortunately, some miss the point and do not appreciate the originality nor the founding principles of computing and claim, for example, that "sometimes they do not iterate", like nature. Of course, there may be hardware problems, but these are problems, usually and easily fixed. Instead, non-iteration, identically, is part of the principles of non linear dynamics. 
Let alone life sciences where the main invariant is... variability, even within "structural stability", which is not phenotypic identity.

But let's go back to the interface mathematics/physics. The passage from the physical process to the formal system is done by means of measurement. If the only formalization/determination we have, or which we consider to be relevant for a given process, is of the deterministic but chaotic type, the (classical) physical measurement, which is always an interval (and which we describe, in general, within a context of continuity) enables to only give an interval as input for the computation. And this has a further fundamental connection with physics, that we already mentioned: the interval topology yields the topological invariance of dimension, a fundamental property of the continua of mathematical physics. Now, given that non-linear dynamics are mixing (the extremes and the maximum and minimum points of any interval are "mixed" at each step) and have an "exponential drift" as Turing puts it. This is a nice way, Turing's way, to say what we observed: the interval of measurement soon occupies in a chaotic - mixing - way an increasing part of space and it is impossible to further predict the evolution of the physical process. If we were to use as input not an interval, but a rounded value, this would obviously not help prediction: the result of the computation may have nothing to do with the physical evolution - for the logistic function, with $k=4$, a rounded value at the 16 th decimal makes any physical process unpredictable approximately from the 50th iteration, - this is calculated using the value of the Lyapounov exponent, [12].

To return to the baker's dough, a very simple and common example, it is a physical process determined by a demonstrably chaotic evolution function: it is a mistake to say, as we sometimes hear, that it is non-deterministic; it is unpredictably deterministic, which is quite different (the error, in this case, is exactly Laplace's error, for whom determination should imply predictability). In physical terms, the forces at play are all known, the "tent" function determines its evolution well, just as the logistic determines that of the agoantagonistic processes or as the equations of Newton-Laplace determine the evolution of Poincaré's three bodies. In classical physics everything is deterministic, even a toss of dice! But sometimes, it is impossible to predict or calculate evolutions because of the approximation of physical measurement in conjunction with the sensitivity to contour conditions, proper to the intended, modellizing, mathematical systems (or with the excess of relevant but hidden variables in the process: Einstein hoped to transfer this very paradigm to quantum physics).

So, in general, the mathematical systems which we write are computable and predictable; some of these systems, being chaotic, refer to unpredictable 
physical processes. In principle, the latter, as such, do not "compute", in the sense of the Church thesis or of its continuous versions. Let's specify this point once more.

Computation is an issue of numbers, in fact of the (re-)writing of integer numbers: lambda-calculus, Turing Machines, are actually a paradigm of it. Now, to associate a number to a physical system, it is necessary to have recourse to measurement, a challenge and major issue regarding principles in physics, as has been realized since Poincaré and Planck, extraneous to the logic of arithmetic and, thus, largely forgotten by computationalist (the World is a large "digital computer"). Classically, if we were to decide that a certain state of a physical process constitutes the input, and another the output, and that we associate these states to measurement intervals and if all we know of this process is mathematically unpredictable, then it will be impossible, in general and after a sufficient amount of time (if time acts as a control parameter), to compute or predict an output interval from the input interval of the order of magnitude of the given physical measurement. In short, if we launch a good old physical double pendulum, if we manipulate a baker's dough, it will be impossible to compute, within the limits of measurement, its position after 5 or 6 oscillations or foldings, although they may themselves be determined by two equations or by an evolution function in which all is computable. So the double pendulum, the stretched dough, as a physical machine/process, does not compute a computable function. As for quantum mechanics, we will return to this below.

But do they define a function? Because in the same initial (physical) conditions, they do not generally iterate, and therefore do not even define a mathematical function of an argument (which one?) within the initial interval of measurement, a function which would always return the same value! It would therefore be necessary to parameter them across time according to a physical reference system: at best they would define a function with multiple variables of which one is the time of the chosen reference system. This makes them rather useless as machines for defining non-computable functions: they cannot even be re-used, in time, to compute the same function, because at each different moment we would have different values which are a priori non-repeatable. And no one would buy them as "non-Turing" machines.

And here we are confronted once more with another common error: expecting that if the physical Church thesis was to be false, then the counter example should return a process which computes more than Turing. But such is not the case. This is an error because a "wild" physical process (as biologists would put it), in general, does not even define a function, that is, a single-valued argument/value relation. The very idea that a process 
could be reiterated suggests that it could be redone in the same (identical, as within a discrete framework) initial conditions. And this, which is so trivial (in both the English and French senses of the term) for a discrete state machine, is unachievable in nature, except in very rare or artificial cases, save the extension of the parameters to an additional temporal dimension which takes account of the counting of the experience performed. In what concerns life phenomena, do not by any means try to make the halting of a Turing Machine computed by a paramecium and the movements of its two thousand cilia: quite upstream to computation, paramecia do not define functions by their activities (between the paramecium and computation there is the "wall" of measurement: how to measure, what to measure, using which level of approximation?).

Quite thankfully, we have invented an alpha-numeric machine that is not wild at all, but well domesticated and exact. It comes with its own reference system and clock (hence the problem in concurrent networks, where a spatio-temporal absolute is lacking). Thanks to its structure as a discrete state machine, as Turing emphasized from the moment he produced his invention $^{5}$, this machine enables an access to the data and computations and ... it iterates, identically, when made to: there are the two reasons for its strength. And even within computer networks, thanks to the discrete aspect of databases, we manage to iterate processes, as we said, despite the challenges entailed by concurrency within physical space-time.

\section{Return to COMpUtability in MATHEMAtics}

Let's return to the issue of computability beyond the measurement which we just addressed.

Mathematically, chaos is a long-time phenomenon: as for the sensitivity to the initial conditions, it is the long-time asymptotic behaviour which differs between chaos and integrability. What is the evolution of the baker's dough in the case of an infinite number of iterations? Let's be more specific and look at the case of ergodicity, a property of chaos which is actually weak (and noncharacteristic). A system is ergodic when, for almost all points (the "ergodic" ones), the temporal and spatial averages of any observable coincide at the infinite limit. This is a property "in measure" (measure meant here in the mathematical sense) and it requires, in its "time" component, an integration over an infinite time.

\footnotetext{
${ }^{5}$ Or shortly after: in 1936, it was nothing more than a logical machine, "a man in the act of computing"; it is only after 1948 that Turing viewed it also as a physical process a discrete state one, as he called it in [30] and [31].
} 
Clearly, the question of computability of average up to time $t$ for any value of $t$ makes sense, and has a clear answer in terms of properties of computability of ordinary differential equations, but the passing to the limit $t \rightarrow \infty$ shifts us towards these limits of which it was question earlier and which we will return to now. In particular, the rate of (mathematical) convergence will intervene in the answer to the first question, and obtaining information on the rate of this convergence is a very delicate problem especially in what concerns real, practical flows, those which nature provides us with.

One must nevertheless not forget the huge contribution of computer science: the computer, however fundamentally non-chaotic, "sees" chaos perfectly well, suggests it, presents it to our eyes in a very spectacular and now completely indispensable way. And this by the (approximated) images of the density of trajectories, by the amazingly different results in the change of the 16th decimal or so etc.. By developing turbulences of any sort in an otherwise unfeasible way and showing them on a screen, a fantastic help to scientific insight is achieved.

\section{The passing to continuity}

The passing from rational numbers to real numbers poses more problems than it may seem: a quantum system in a finite volume is indeed represented by a vector space of finite dimension. Yet, some caution is required; not only must this space be bounded, but so must the momentum dimension, that is, the phase space, of which the standard of measurement is Plank's constant. But the superposition principle immediately makes the number of states infinite (to the power of the continuum): this is precisely the "vectorial aspect" of the theory. Quantum mechanics resides in vector spaces and the "finitude" of space entails the finitude of the dimensions of these spaces, not their cardinals. It is impossible, for a set value of the Planck constant, to put anything but a finite number, $d=\frac{V}{\hbar}$, of independent vectors (states) within a finite volume $V$, but thanks to (because of) the superposition principle, it is in fact possible to put an infinite number of vectors, as many as there are points in $\mathbb{C}^{d}$. This doesn't mean of course that, for certain definitions of information, the "quantity" of information could not remain bounded as the system remains confined in a finite volume, but this shows the difference of the concepts of space in classical and quantum situations (for a discussion of this discrete/continuous dichotomy see e.g. [23]).

One must then evoke the Rolls-Royce of mathematical physics: the theory of partial derivatives equations (PDEs). A PDE can be seen as an ordinary 
differential equation in infinite dimension, it is like a system of ordinary differential equations each of them labelled by a continuous parameter (by the way, it is precisely this aspect which the computer retains before discretizing this continuous variable): each point in space "carries" a dynamic variable of which the evolution depends on immediately (even infinitesimally) neighboring points. Contrary to ordinary differential equations which, in general, have a solution for all values of time, we can say that a PDE has (still generally speaking, in the "hyperbolic" case) a limited life span, in the sense that its solution can explode in a finite amount of time. We therefore witness the emergence of two pitfalls: one passing to infinity for space, and one passage to "finitude" for time. This is another example where the very notion of computability does not apply well to the physico-mathematical phenomenon.

Let's now ask ourselves why chaos was invented. The sensitivity to the initial conditions has appeared as a negative result, preventing integrability. The negation of integrability aims to be perceptible in a finite amount of time (since integrability places us in front of eternity). But it is very difficult to demonstrate that a system is not integrable. A recourse to asymptotic results is then made (a bit like the use and abuse of statistics today), these often being the only available ones. Hence the intervention of chaos, another extreme and antipodic point of integrable systems, and hence its limits with regard to computability.

\section{Non-DEterminism?}

In computer science, we often define non-functional relations as being "nondeterministic"; in short, when we associate a number to a set. Let's first examine the case of so-called "non-deterministic" Turing Machines, of which the transition functions have precisely this nature (from a value to a set of values). Calling them non-deterministic may be reasonable, as an a priori as long as we remain within logico-computational formalisms, but makes no physico-mathematical sense. Is there an underlying physical process which will associate to an input number a set or an element of the set in question? Not necessarily. So, if deterministic (classical) means (potentially) determined by equations or evolution functions, a "non-deterministic" Turing Machine is indeed determined by a function which associates an output set to an input value (an issue of asymmetrical typing, nothing more). If there is indeed a choice of value among a set, quantum physics could certainly propose one: it is legitimate to say that quantum measurement, by giving probabilities within possible values, performs such an operation. Can we use a classical process for the same association? Why not: we can take a physical double pendulum, determined by two equations or the baker's 
dough, of which the evolution is described by the "tent" function - so there is nothing more deterministic than these two objects and their evolutions. We give them an input number; the evolution starts off on an interval of measurement which is roughly centered around this number, but the result, which is unpredictable after a few iterations, can take a value among all those within the space. This is what deterministic unpredictability is. Yet, with a playful use of language (and a little bit of confusion), we could also say that this association (one value/one set) produces a non-functional relation and so consider it as non-deterministic. But contextual clarity, necessary to the good relationship between mathematics and physics, then disappears: all is grey and that which is not functional (nor calculable) is the same, as there is no more difference between classical unpredictable determination and quantum indetermination, typically.

In what concerns concurrent systems, the situation is more interesting. Over the course of a process, which occurs within physical space-time, choices are made among possible values, following the interaction with other processes. In concrete machines, these choices can depend on classical, relativistic, quantum, or even human phenomena which intervene within a network. In the first two cases, everything is deterministic, although described by nonsingled-valued relations and although there may be classical unpredictability (which value within the determined set? - a lesser temporal discrepancy can produce different choices). In the other two cases (quantum and "human"), the choice of value will be intrinsically non-deterministic, but, in principle, for different reasons (not being able to give an appropriate physical name to the will of humans acting upon a network). In some cases, authors in concurrency, by non-determinism, refer to a "do-not-care" of the physical "determination": whatever is your hardware and your (compatible) operating system or compiler, my program for the network must work identically. A new concept of "non-determination" a very interesting one, probably with no analogy in natural sciences (my soul doesn't work independently of my body, this was Descartes's mistake, nor it is portable).

It would be preferable to introduce a notion of "indeterminacy" specific to computer science corresponding to the absence of univocity of the inputoutput relation with choices, in particular that which can be found in "multitasking", in the concurrence of network processes, etc.

\section{The discrete and the "myth" of continuity.}

This loss of meaning of continuous physics can be found in Gandy's reflections on Church Thesis, for instance (he was one of the pioneers of the physical Church thesis, [17]). He posits among other things a physical world 
within which information is finite, because it is part of a finite universe. So it is made to be discrete, all the while remaining within a classical framework, and then deterministic chaos disappears, as happens with the Turing Machine (Turing says this very clearly in [30], see also [21]) - see also the discussion on finitude in quantum mechanics in the preceding paragraph.

Firstly, the mathematical definitions of chaos use continuity (to represent the interval of measurement); they will loose their meaning when the natural topology of space considered is discrete topology (we keep returning to this, because it is important: the access to the measurement of the process will then become exact, because isolated points are accessed, mathematically another way to summarize all which we have just said).

Now Gandy does not appear to have followed his master Turing, the inventor of the "Discrete State Machine" (which is theoretically predictable, says Turing, [30], though it may be practically hard to predict - very long programs), in the adventure of the continuity of non-linear dynamics (theoretically unpredictable, Turing remarks, this being their most interesting property, [31], see [21] for a discussion).

Now Turing had a good understanding of this issue in the later years of his life, making a remarkable contribution to the development of what he called "continuous systems" (the name which he gives to the linear and non-linear models of morphogenesis, [31], and which he already uses in [30] in contraposition to his machine). In fact, continuity is currently the best tool we have for addressing classical determination. It is the "myth" of an underlying or abstract space, a mathematical continuum, which leads us to think that any classical trajectory is deterministic: it is "filiform" (widthless) and stems from a Euclid-Cantor point (dimensionless, said Euclid). It is a "myth" in the sense of Greek mythology, because it constructs knowledge, but removed from the world. This limit, the point and Euclid's widthless line are not given by measurement, our only access to the physical world. The myth is at the limit, like the thermodynamic integral which gives us the irreversibility of diffusion at the infinite limit (that is, which demonstrates the second principle, by supposing an infinity of trajectories for the molecules of a gas within a large, but finite volume). The mythical (conceptual, if the reader prefers) limit makes us understand: how audacious this beginning of a science, this imagination of the widthless line, of the point of no dimension. Without those limit (infinitary!) concepts, which are not in the world, there would have been no theory of the measurement of surfaces: it is necessary to have "widthless" edges and dimensionless points at the intersections of lines to propose a general theory of areas (how thick should otherwise be the 
border of a triangle?). Finitude, as the discrete of a naive and pre-scientific, pre-Greek perception, entails machine-like stupidity.

In this context and since Einstein, we have gone further and have even come to say that finite, for the universe, does not mean limited. Think of the relativistic model of the Riemann sphere: it is finite but unlimited, contrary to the notion of finitude as limitation to be found with Euclid (infinite $=$ apeiron $=$ without limits). Why would the information on the Riemann sphere be "finite" in such a model? Of which type of finitude would we be speaking of? Euclidean finitude or modern unlimited finitude? Be it relativistic or quantum, "finitude" contains infinity.

Except for great thinkers such as Turing, logicians and computer scientists tend to have a culture of the finite/discrete/Laplacian, as Turing said of his machine, which is difficult to escape. Its origin is the arithmetizing perspective of Frege with regard to the "delirium" of Riemannian geometry and the incomprehension of Hilbert, one of the great figures of mathematical physics, concerning unpredictability, of even Poincaré's type of undecidability (it is impossible to calculate - decide - the position of three planets after a sufficiently long period of time), when he speaks of mathematics: 20 years later, he will launch one decidability conjecture after the other, all of them being false (Arithmetics, Choice, Continuum Hypothesis), despite the highly justified objections from Poincaré (Mr. Hilbert thinks of mathematics as a sausage-making machine!). Poincaré had already experimented with undecidability, as unpredictability, though in the friction between mathematics and physics (not of purely mathematical statements, Hilbert's question). However, this culture of predictable (and integrable!), of the determination, within a universe (a discrete, finite and limited database), has given us marvelous Laplacian machines. Let's just make an effort to better correlate them to the world, today. A good practice, and theory, of modelization and of networks, that of concurrency, impose them. They evolve within a space-time which we understand better, for the moment, thanks to continuity. Thankfully, there are also hybrid systems and continuous computability which also propose quite different perspectives. And likewise for the work of Girard which tries to enrich logic with concepts that are central to the

field of the physico-mathematical: symmetries, operator algebra, quantum non-commutativity.

But let's return to quantum mechanics.

\section{The CASE OF QUANTUM MECHANICS}

The quantum issue could at first glance present a perfect symbiosis between the two preceding sections: we are dealing with a fundamentally fundamental 
equation, Schrödinger's equation, which derives from nothing, which must be at the center of any fundamental process, and of which the mathematization is perfect, depending on only a single parameter (actually, is Planck constant's value a computable number?). Moreover, "measurement" takes on a whole new dimension. The interval, as such, no longer exists and intrinsic randomness is introduced.

Let's now mention the importance, particularly in the field of the physics of elementary particles, of the role played by computers. The computation of precise numeric values, for instance the calculation of the electron's magnetic moment, and their literally "phenomenal" concordance with experience has doubtlessly had a crucial importance for the development of the theory. And this precisely in the very field where computers have become irreplaceable: numeric computation. Associating a number to hundreds, to thousands of Feynman diagrams is an operation beyond human capability and which computer science bravely accomplishes.

The results provided by quantum physics are precise, and have a level of precision which any other physical theory has yet to attain. They are also discrete, meaning that the richness of continuity has been lost, and that we are facing a (discrete) play of possibilities. Of course, what we are actually measuring is a classical object, a classical trace (bubble chamber, photographic plate...) with a quantum value. We are indeed at the heart of the problem: a quantum measurement provides values belonging to a discrete set (set of values specific to the Hamiltonian), hence a certain rigidity that is a source of stability and therefore of precision (those of discrete topology). Seen from this angle, quantum "precision" seems tautological in a way; we allow ourselves no leeway around discrete values which would enable to extend into the voluptuousness of imprecision. We could even say: let's provide ourselves, once and for all, with all the values specific to all the Hamiltonians of the world and we will have a field of "outputs" which is discrete in its very essence.

But this is precisely forgetting that the result of such measurement is obtained upon a classical object given that it is accessible to us. The spectral lines appear on a photographic plate. And we therefore find the classical continuum a posteriori, and its virtues which are harmful because prone to introduce imprecision. And so what the fact that quantum mechanics is incredibly precise signifies is that during experiments, it leaves classical traces of an extreme level of precision, practically exhibiting a discrete structure of continuity.

And this is not tautological at all. 
In addition to this discreteness, and precision, quantum mechanics has caused some difficulties by conferring a random aspect to the result of measurement. Let's say right now that something had to happen, because the principle of quantum superposition prohibits a direct access, beyond measurement, to the quantum space of states (we do not "see" superposed states, or entangled states); more accurately, we "look" at them, and they must be looked at to be seen, by getting measured, they "de-superpose" themselves, they de-entangle. This random aspect immediately escapes any computational system of ... computability. No more determinism, no more equations. Of course, it is possible to talk about statistics, and to wonder whether these statistics are computable. We then return to the non-deterministic algorithms of the preceding section, but with a different problem.

Quantum algorithms are a perfect illustration. Let's recall that a quantum algorithm consists in a quantum system evolving from an initial piece of data having, in a way, a classical "input". By principle of superposition, entanglement, at the end of an evolution, has done its job and the final state is typically quantum, superposed in several states, of which a single one contains the "output" sought. To get it, we then perform a measurement that is supposed, by construction, to produce the good result with a maximal probability.

What is Turing computable in all of this?

We can wonder regarding the first part of the quantum evolution related to quantum "equational" evolution, modulo the remarks made at the end of the section 6 concerning PDEs (Schrödinger's equation is a PDE after all, but a linear and not an hyperbolic one), and could possibly answer : yes, this part of the quantum evolution is computable. But the last phase, that of measurement, again escapes computational reduction: the random aspect of measurement, let us rest assured, will never enable a quantum computer to decode a credit card at the desired moment with certainty.

\section{Quantum algorithms versus non-deterministic algorithms}

It could be advisable to specify the important difference between quantum and non-deterministic algorithms, a source, it appears, of many confusions. Indeed, one could confuse two very different "parallel" aspects.

A quantum algorithm, in a way, works well in parallel; computation is fundamentally vectorial because of the very nature of quantum dynamics. But the final result, that which needs to be extracted from the final quantum state, is a single one of the components present within the latter. The other components, the whole "final state" vector, has no interest as such: firstly because it is inaccessible, then because the other components (other than the 
component containing the results) do not carry any information related to the initial problem. So it is not an issue of dispersing the information in order to parcel it out and hence increase the power of the computation and then "patching the pieces back together", in a way, but rather of placing oneself within a space (a quantum space, and again, one that has not yet been satisfactorily achieved experimentally) from which one needs to suddenly return in order to finish the computation.

Because the essential is indeed there: the "computation", the "process" is finished only once the ultimate measurement is taken. It is this total process which must be placed in the view of computability, and not the purely quantum part which conveys no information. It is exactly the same idea which is responsible for there being no "EPR paradox" because, although we are acting from a distance upon the entangled vector, no information is transmitted.

Let us mention also that logic based on quantum mechanics paradigm has been recently introduced by J.Y. Girard, without explicit motivation in the direction of quantum calculus [18]. We conclude by saying that the randomness of quantum mechanics is intrinsic, it escapes computation. What about classical randomness?

\section{RANDOMness, BETWEEN UNPREDICTABILITY AND CHAOS}

In [4], classical randomness and deterministic unpredictability are identified, from the point of view of mathematical physics. Randomness would present itself, we observed, at the interface between mathematics (or, more generally, between language) and physical processes. It must however not be ignored that, in certain probabilistic, purely mathematical frameworks (measure theory), we can also speak of randomness, away from physical processes. By computation theoretic tools, Per Martin-Löf advanced, 40 years ago, a purely mathematical notion of randomness. More specifically, one can, by means of computability, tell when an infinite sequence of integers (of 0s and 1s for example) is random, without reference to an eventual physical generative process. In short, a random sequence is Martin-Löf computable if it is "strongly" non-computable, a definition which requires a little bit of work (see [27] for a recent overview). In a sense, formal computability/predictability can tell us when we leave its domain: a bit like Gödel who, in his proofs, never left the formal, and who was yet able to give a formula which escapes the formal (which is formally unprovable, jointly to its negation).

Moreover, what interests us here, this purely mathematical randomness, is "at infinity", exactly like the randomness within chaotic classical dynamics 
is asymptotic: a random Martin-Löf sequence is infinite (the initial segments are at best incompressible).

What can then be said of the relationship between this notion, purely mathematical, and physics? From the statistical viewpoint, which was the preoccupation of Martin-Löf (ML) at the time, every thing is fine: the distribution of the probabilities of a ML-random sequence, for a good probability measurement, is that of the toss of a coin, to infinity. But what about the relationship to the physico-mathematical of dynamical systems? How can one pass directly, by mathematical means, without reference to the physical processes that the two approaches modelize, from ML-randomness to unpredictable determinism (systems of equations or evolution functions)? We can see possible correlations in some works which are in progress (particularly those by M. Hoyrup and C. Rojas in the CIM team of one of the authors): the points and the trajectories within chaotic systems are analyzed in terms of ML-randomness, all the while using suitable notions of measure, of mathematical entropy and Birkhoff ergodicity. In the two cases, those of sequences of integers and of continuous dynamics, we work to infinity.

Let's be more precise. A dynamical system, as a purely mathematical formalism for physics, is said to be "mixing" if the correlation of a given pair of observables decreases at least polynomially with time. Like ergodicity,this is an asymptotic property of "disorder", a weak forms of chaos. What was recently proved is that, in mixing dynamics, ergodic points coincide with ML-random ones (in fact for a slightly different definition of MLrandomness). Thus deterministic unpredictability, as ergodicity in mixing dynamics, overlap with a strong form of undecidability. In other words, if we want to relate physical processes to effective computations, which is an issue of elaboration of numbers, we can, but, at the limit, all processes that are modeled by a somewhat chaotic system, produce non-computable sequences, within the mathematical system. Or, also, (strong) non-computability (as ML-randomness) may be found in formal writings of the physical world (dynamical systems are perfectly formalisable, of course). That is, at the limit, we may say "no" to Laplace's conjecture of predictability of deterministic systems and, this, in terms of undecidability, à la Gödel. Predicting, in physics, is a matter of "saying" (pre-dicere, to say in advance) by a formal language or system about a physical process in finite time, as we said several times: by these results, instead, Poincaré's finite unpredictability joins 
undecidability, asymptotically. In conclusion, deterministic ergodic and mixing dynamics, which model somewhat "chaotic" physical processes, generate non-computable features ${ }^{6}$.

\section{General Conclusions}

The reader might have felt that the authors have a point of view "against" a vision of the nature that would be too much organized around computations. Once again computers have brought so much to science that it is not necessary to recall the benefit provided. It seems to us that this situation, where a given viewpoint invades a whole field of science happened several times in the past. An example is the case of mathematical analysis at the turn of the last century, a period where many new objects in mathematics were born, such as functions nowhere differentiable, Cantor sets, summation methods of diverging series .... To focus on the latter let us quote Emile Borel, in the Introduction of his famous book on diverging series [8], where he talks about the fact that analysis "à la Cauchy", based on convergent Taylor expansions of analytic functions, although it brought a considerable amount of progress in mathematics, fixed also into rigidity a lot of nonrigorous methods used by the geometers (in the sense of physicists) of the older time: "This revolution" was necessary: nevertheless one might ask if dropping the less rigorous methods of the geometers (...) was good or not : (..) but this period ${ }^{8}$ being passed, the study of former methods might be wealthy...".

Let's see what we have done so far. We have reviewed certain aspects of computation in physics and in mathematics. We have seen that many situations in physics, even classical physics, cause processes which are "beyond computation" (in the sense of "calculus resolving equations") to intervene. We have also mentioned the calculatory contribution of computer science and its essential role. Now, let's not forget the importance as such of the plurality of "visions" for understanding the natural sciences, a plurality which has always existed in the sciences. The new perspective proposed by the discrete, in great part due to the contribution of computer science, is a conceptual and technical resource, which adds itself to the differentiable physicomathematical continuum, from Newton to Schrödinger (or even consider, for

\footnotetext{
${ }^{6}$ see [16], [15] and http://www.di.ens.fr/ longo/ for ongoing work. Connections between algorithmic and quantum randomness are analyzed in [11].

${ }^{7}$ The Cauchy and Abel rigorous vision of Analysis based on convergence of expansions of Taylor series.

${ }^{8}$ of rigor
} 
example, its importance of computer modelling in biology, to mention another discipline, [32]). On the other hand, the reduction to a conceptual and mathematical dimension that is too "computational" (in the excessively naive sense of the term) would, in our view, lead us to sterile boredom, in which even the "nuances" of the post-Laplacian continuum would be absent. Finally and in particular, within "equational" framework for the play between the continuum and the discrete, we have discussed notions that appear to be fundamental to modern science, such as those of determinism and of predictability, from where emerges the notion of uncertainty. But let's take a further look.

As compounded in [4], classical physical randomness is of an "epistemic" nature, whereas that of quantum measurement is intrinsic or "objective": a distinction which should be solely an instrument of clarity, of conceptual clarity if possible, and nothing more. By this we refer to several aspects among which the one of interest to us is the following: classical randomness can be analyzed by means of different methods. In short, it is possible to address dice, the double pendulum, the baker's dough, etc., in terms of statistically random sequences and of probability distributions (central limit theorem, etc.), but also by means of the mathematics of chaotic determinism (if we have the courage to write the several equations needed for the movement of dice, it is easy for the double pendulum and the baker's dough). Some people, mainly in the field of computer science as we have seen, say that the toss of dice or that the baker's dough (or even the three bodies?) are non-deterministic because, by using the approximation of measurement, it is possible to associate several numeric outputs to an exact input number and the same wording is used for computational non-determinism. It is an abuse of language which ignores the specifications brought by the broadening by Poincaré of the field of determination, which includes classical randomness in the field of chaotic determination (the non-linearity of "continuous systems", says Turing), and by the indetermination of quantum physics. This is specific to the culture of the discrete, which is wonderful for our discrete state machines, but which misses the 120 years of geometrization of physics (geometry of dynamic and relativistic systems) and which fails to appreciate the role of measurement (classical/quantum).

We thus see the apparition of three idealizations thanks to which we could think it possible to discover and understand the world (classical).

1. The digital, discrete ideal which (possibly) shows nature as computing and only as computing. Computing, iterating and reiterating to infinity with a wonderful and misleading precision. 
2. The ideal of continuous mathematics, where nature (mathematics) solves equations. In itself, this vision is perfectly deterministic, the equations have solutions.

3. The ideal of the equation, for which nature divides itself into different scales, impenetrable the ones into the others-for example the quantum world, the classical one, hydrodynamics, celestial mechanics, cosmology etc.

These ideals $(1,2,3)$ are placed in anti-chronological order: historically, equations were the first to appear, followed by their mathematical models and finally by their digital simulation.

To conclude, let's look at the connections and anti-connections between these three worldviews, these three tiers that we could compare to Girard's three basements. This would be the result of the present work.

At first glance, we could easily go up from the 3rd to the 2nd and then to the 1st level. Continuous mathematics seem perfect for equations, and digital approximation has become so commonplace that one must almost hide to criticize it. But the elevator does not work properly: between the 3rd and 2nd levels, Poincaré shakes things up (the sensitivity to the initial conditions, as it is, makes difficult the practical idea of a trajectory within continuity), and between the 2 nd and 1 st levels we have lost, by climbing to the level of the discrete, a few aspects that were important to continuity (the fluctuations below the threshold of discretization as well as... the discrete blackness of milk). If we take the stairs to go down, we get dizzy: lack of computational equivalency for the passing to continuity, and loss of reliability with the introduction of the interval of imprecision when passing from the 2nd to the 3rd levels...

And there is quantum mechanics with its intrinsic randomness. Ideal \#3 is then shattered during measurement: no more equations. Of course, physics can make do without individual measurement phenomena: we have not (yet) experimentally observed the reduction of wave packets during unique events, all which we can observe is averages, statistics. But recent physics pushes towards the study and observation of simple quantum physical systems which are always better at conducting the "gedenken Experiment" ${ }^{9}$ of the founding fathers [26], and in any case the reduction of the wave packet during measurement is, we believe, a necessary component of quantum formalism, an axiom which makes it coherent.

This situation is not new in physics: we do not observe Newtonian mechanics in a mole of gas. And yet it is thanks to such mechanics that we can reconstruct the dynamics of gases and thermodynamics, which are the result of the passing to infinity from a finite non-observable model.

\footnotetext{
${ }^{9} \mathrm{EPR}$ paradox, Schrödinger's cat for example.
} 
Acknowledgements

We would like to thank to Eugène Asarin, Olivier Bournez, Mathieu Hoyrup and Cristobal Rojas for their critical reading of the manuscript. The authors' papers may be downloaded from http://www.di.ens.fr/ longo/ and http://www.dma.ens.fr/ paul/.

\section{REFERENCES}

[1] Aceto L., et al. (eds.) The difference between Sequential and Concurrent Computations. Special issue, Math. Struct. in Computer Science, Cambridge U. Press, n. 4-5, 2003.

[2] Arnol'd V.I., Equation différentielles ordinaires. MIR, 1976.

[3] Bailly F., Longo G., Mathématiques et sciences de la nature. La singularité physique du vivant, Hermann, Paris, 2006

[4] Bailly F., Longo G., "Randomness and Determination in the interplay between the Continuum and the Discrete". Mathematical Structures in Computer Science, 17(2), pp. 289-307, 2007.

[5] Baverman M., Yampolski M. "Non-computable Julia sets". preprint, 2005 (http://www.math.toronto.edu/yampol/).

[6] Bell J. textbfA Primer in Infinitesimal Analysis, Cambridge U.P., 1998.

[7] Blum L., Cucker L., Shub M., Smale S. Complexity and Real Computation. Springer, 1998.

[8] Borel E., Leçons sur les séries divergentes, Gauthier-Villars, Paris, 1928.

[9] Bournez O.. Modèles continus. Calculs. Habilitation DR, LORIA, Nancy, 2006.

[10] Bush V., "The differential analyzer". Journal of the Franklin Institute, 212, 1931.

[11] Calude C., Stay M. "From Heisemberg to Gödel via Chaitin", International J. Theor. Phys. 44 (7), 2005.

[12] Devaney R. L. An Introduction to chaotic dynamical systems, Addison-Wesley, 1989.

[13] Dowek G. "La forme physique de la thèse de Church et la sensibilité aux conditions initiales", proceedings of the Cerisy colloquium, September 2006 (Joinet et al., eds), Hermann, 2008.

[14] Feynman R., Quantum mechanics and path integrals. McGrawth-Hill, 1965.

[15] Gacs P., Hoyrup M., Rojas C. "Schnorr randomness-A dynamical point of view". In preparation, 2008.

[16] Galatolo S., Hoyrup M., Rojas C. "Computable dynamical systems, SRB measures, random and pseudo-random points", in preparation.

[17] Gandy R., "Church's Thesis and the principles for Mechanisms" in (Barwise et al. eds) The Kleene Symposium, North Holland, 1980.

[18] Girard J.Y., Le point aveugle, Hermann, Paris, 2007.

[19] Hainry E., Modèles de calculs sur les réels. Thèse, LORIA, Nancy, 2006

[20] Hoyrup M., Kolcak A., Longo G. "Computability and the Morphological Complexity of some dynamics on Continuous Domains". Invited survey Theor. Comp. Sci., to appear, 2007.

[21] Longo G. "Laplace, Turing and the "imitation game" impossible geometry: randomness, determinism and programs in Turing's test". In Epstein, R., Roberts, G., 
\& Beber, G. (Eds.) The Turing Test Sourcebook. Dordrecht, The Netherlands: Kluwer, 2007.

[22] Moore C. Recursion theory on reals and continuous-time computation. Theoretical Computer Science, 162, 1996.

[23] Paul T. "Discret-continuous and classical-quantum", Math, Struct. in Computer Science, 17, p. 177-183, (2007).

[24] Paul T. "On the status of perturbation theory", Math, Struct. in Computer Science, 17, p. 277-288, (2007).

[25] Pour-El M.B., Richards J.I., Computability in analysis and physics. Perspectives in mathematical logic, Springer, Berlin, 1989.

[26] voir l'exposé de J-M. Raimond au colloque "Qu'est-ce qui est réel?" (organisateurs C.Debru, G. Longo, T. Paul et G. Vivance). École Normale Supérieure, 2005, http://www.diffusion.ens.fr/index.php?res=conf\&idconf $=807$.

[27] Rojas C. "Computability and Information in models of Randomness and Chaos", Math. Struct. in Computer Science, vol. 18, pp 291-307, 2008.

[28] Shannon C., "Mathematical theory of the differential analyzer". In J. Math. Phys.. vol. 20, 337-354, 1941.

[29] Turing A.M., "Intelligent Machinery". National Physical Laboratory Report, 1948. In Meltzer, B., Michie, D. (eds) 1969. Machine Intelligence 5. Edinburgh University Press.

[30] Turing A.M., "Computing Machines and Intelligence", Mind, vol. LIX, n. 236, p. 433-460, 1950.

[31] Turing A.M., "The Chemical Basis of Morphogenesis", Philo. Trans. Royal Soc., vol. B237, p. 37-72, 1952.

[32] Varenne F., Du modèle à la simulation informatique. Vrin, Paris, 2007.

[33] Weihrauch K., Computable Analysis. Texts in Theoretical Computer Science. Springer, Berlin, 2000. 